\title{
Pain Management for the Young Adult Rheumatology Patient in an Era of Medicinal Marijuana Legalization
}

The young adult (18 to 23 years old) rheumatology patient has unique needs. While transitioning through a life stage that encompasses physical and emotional changes, these patients are also dealing with the challenges of coping with the many facets of chronic illness. Pain, a prevalent symptom of rheumatic disease, impairs physical, social, and emotional function, requiring a pain-directed treatment plan in parallel with disease modification. In the current context of the medical legalization of marijuana (herbal cannabis) worldwide, many young patients may be looking to this compound as a pain management option. Familiarity with marijuana as a recreational product that is socially acceptable for many youth may encourage therapeutic use, smudging the lines between recreational and medicinal use. Therefore the medical community must be knowledgeable about current evidence of benefits and risks of marijuana as a therapeutic option to competently advise these young people.

\section{Traditional Principles of Pain Management}

Principles of pain management, generic to all pain conditions, should incorporate nonpharmacologic measures as a first step, with attention to good lifestyle habits, education to strengthen the therapeutic alliance, and promotion of coping strategies ${ }^{1}$. Without providing a detailed description of current pharmacologic treatments for chronic pain, any choice for the young adult must especially take into consideration effects on cognition, potential effects on the developing brain, and interactions with other substances. The pharmacologic treatment of pain should begin with use of the simple analgesics and thereafter with nonsteroidal antiinflammatory drugs (NSAID). Compliance with a regimen of continuous medication, especially with more frequent dosing as for some NSAID, remains a challenge. While not denying the need for pain management, the evidence to support opioid use to treat rheumatic pain is scant. Opioids should therefore be reserved for those with severe pain unresponsive to standard measures, and ideally prescribed for short time periods. Opioids are addictive. They affect cognition, psychomotor control, and mood, and they pose a particular concern if used for psychoactive effects rather than analgesia ${ }^{1}$. Taking into consideration increased opioid abuse, including the risk of death related to prescribed opioids, extreme caution must be exercised when treating young people.

\author{
Groundswell of Medicinal Marijuana Use in Rheumatic \\ Conditions
}

Cannabinoid molecules hold promise for pain relief. To date there are 2 pharmaceutical oral preparations, dronabinol and nabilone, with US Food and Drug Administration approval for the treatment of chemotherapy-induced nausea, but not for pain relief. Dronabinol is a stereoisomer of tetrahydrocannabinol (THC), and nabilone is a synthetic analog of THC with fewer psychoactive effects. A third preparation is an oromucosal spray of nabiximols, a combination of $\Delta 9$-THC and cannabidiol (CBD); with possibly fewer of the psychoactive effects attributed to CBD. Medicinal marijuana, which contains an abundance of cannabinoid and noncannabinoid molecules, is legal in Canada and in more than 20 American states; it is also popularly advocated as a treatment for rheumatic pain ${ }^{2}$. Its legalization fosters the perception that marijuana is harmless and leads to widespread use.

Notwithstanding an abundance of neurophysiologic study, as well as some evidence for use of cannabinoids in other medical conditions, evidence for an effect in rheumatic diseases is sparse ${ }^{3}$. With only 4 small randomized clinical trials in various rheumatic conditions, it is not possible to draw conclusions regarding effects ${ }^{3}$. A single study examined the use of nabiximols in rheumatoid arthritis; and 3 small studies examined the effects of nabilone in patients with fibromyalgia, without study of marijuana in any rheumatic disease.

\section{Concerns for Marijuana Use in Young Adult Rheuma- tology Patients}

Risks for marijuana use may be categorized as short or longterm effects. The short-term effects are related to cognition, psychomotor, psychological, and vasomotor effects. Impaired cognition and reduced short-term memory may persist for days following marijuana use, with effects on learning and retention of new information. Even in regular marijuana users, acute intake was associated with reduced reaction time, difficulty with selective attention, impaired short-term memory, and motor control that persisted up to 5 hours after consumption, and with increased effects for increased doses ${ }^{4}$. In line with acute effects on psychomotor function and reaction time, acute marijuana consumption is associated with an increased rate of serious injury or death in motor vehicle accidents, and is the most prevalent illicit drug in seriously injured drivers ${ }^{5}$.

Personal non-commercial use only. The Journal of Rheumatology Copyright @ 2016 . All rights reserved. 
The acute psychological and psychiatric effects of marijuana, influenced by the quantities of individual molecules, may differ among individuals. Although generally believed to cause relaxation, other effects that commonly occur include euphoria, anxiety, paranoia, psychosis, lapses in judgment leading to sexual behaviors, and acute withdrawal symptoms ${ }^{6}$. Acute cardiovascular effects related to vasodilatation and tachycardia are recent emerging concerns. The French AddictoVigilance Network identified 35 vascular events spontaneously reported between 2006 and 2010 attributable to marijuana use, with $26 \%$ resulting in death $^{7}$. Although these numbers are small, this report highlights the poorly recognized risk of cardiovascular events associated with marijuana use. Withdrawal and dependence, well documented in animal studies, are identifiable risks associated with marijuana ${ }^{8}$. Withdrawal signs in humans include the physical effect of weight loss and the psychological effects of cravings, anger, aggression, sleep disturbance, hunger, tremors, restlessness, and irritability.

Neuronal development in humans, which is intensely modulated by the endocannabinoid system, continues into the early $20 \mathrm{~s}^{9}$. Exposure to cannabinoids during brain development affects axonal connections, resulting in impaired neural connectivity in areas of the brain important for learning, alertness, executive function, and inhibitory control. Prenatal exposure to cannabinoids is associated with cognitive and behavioral abnormalities that extend into adolescence $^{10}$. Optimum IQ achievement may be affected, especially for regular smokers, and the reward system may be recalibrated to increase susceptibility to abuse of other psychoactive drugs ${ }^{11}$. These neurogenic effects may limit potential lifetime achievement, with a report of lower income, unemployment, criminal activity, and reduced satisfaction with life for young cannabis users in a birth cohort of 1037 persons in Dunedin, New Zealand, followed over 38 years ${ }^{11}$. Marijuana use, especially in adolescence, was associated with neuropsychological decline broadly across many domains and without reversal with cessation ${ }^{11}$.

Smoked marijuana, the most common method of administration, affects respiratory health. The chronic inflammatory changes in the respiratory mucosa are additive with cigarette smoking $^{12}$. Lung cancer risk remains a question. A recent pooled analysis of over 2000 lung cancer cases reported an overall OR for habitual versus nonhabitual or never users as 0.96 (95\% CI: 0.66-1.38), and an OR of 1.73 (95\% CI: $0.75-4.00$ ) for adenocarcinoma ${ }^{13}$. However, in a 40 -year prospective study of 50,000 men, even moderate marijuana use, measured as more than 50 times as a young adult, is associated with a 2-fold increased risk of lung cancer, with persistence of risk when controlled for tobacco and alcohol use and socioeconomic status ${ }^{14}$.

Mental health is an important consideration for young adult rheumatic patients. Chronic marijuana use was associated with 1.4 times higher odds for depression in a study of 8000 US adults, although causality was not established ${ }^{15}$. In view of the increased content of THC in illegal marijuana over the past decade, there have been adverse effects on psychiatric outcomes in mostly recreational users. The risk for psychosis among users is increased, especially if the individual is genetically predisposed, with a relative risk for schizophrenia of 6.7 following at least 50 marijuana exposures by Swedish military recruits ${ }^{16}$. Marijuana may cause dependence and addiction ${ }^{8}$. The nucleus accumbens and the amygdala are brain areas operative in addictive behavior. Marijuana use at least once a week by young people was associated with structural changes of these key brain regions, even when controlled for age, sex, alcohol, and cigarette use ${ }^{17}$. Dependence occurs for $9 \%$ of all users, with this number increasing to $17 \%$ for onset of use in adolescence, and climbing to $25 \%-50 \%$ for daily users ${ }^{18}$. Because exposure can reduce dopamine, users may be predisposed to abuse substances at a later date, particularly if the user is within a social milieu of common drug use.

\section{Guidance for the Healthcare Professional}

Pain management for young adult rheumatology patients has certain idiosyncratic characteristics that can present a challenge, but pain management can be facilitated by knowledge, empathy, and communication. Feedback should be direct and authentic, without sacrificing accountability for empathy. Difficult issues must be addressed in a forthright manner with counterbalance between patient support and patient responsibility. Potential for conflict occurs when possibly harmful self-medication practices through the use of alcohol, marijuana, or other recreational drugs are pursued under the guise of symptom management. These practices may be viewed as a means of pain reduction or a "Band-Aid" for psychological distress, but cannot be condoned.

Specifically regarding marijuana, young patients should be informed that marijuana is not an alternative to standard care of the inflammatory rheumatic disease, nor is it a treatment for mental distress. When there is a request for a prescription for medicinal marijuana, a few steps should be followed $^{3}$. The underlying inflammatory condition must be assessed with exploration of the reason for ongoing pain. Documentation of previous treatment trials, current medications, and all other substances is required. Screening for abuse potential by use of a tool such as the CRAFFT must be done $^{19}$. Examination of the underlying reason for marijuana use is imperative: Is it medicinal? Or is there a component of recreational use? Current function, including work/studies, home and leisure activity, and driving status must be assessed. The patient must know about the uncertainties expressed by the medical community, including lack of knowledge regarding prescriptions, lack of evidence for effect in rheumatic diseases, but compelling evidence for risk $^{20}$.

Pain is a frequent problem for young adults with a

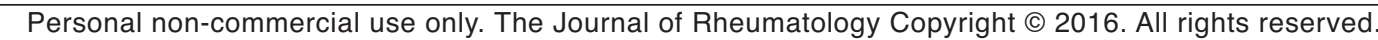


rheumatic disease, with overall outcome influenced by the specific disease characteristics, innate psychological resources, and the social and healthcare network. Although treatment options for disease control are numerous, strategies for pain management remain challenging, with strong emphasis currently placed on nonpharmacologic measures and drug treatment only for limited time periods. Although marijuana is suggested as a therapeutic option for pain management, its negative effects on global health are currently compelling enough to strongly recommend against its use for these patients. Advocacy cannot supersede rational clinical practice and only in extreme situations should marijuana be recommended for the young adult. Perhaps the singular most important facet of coping with pain that can be imparted is optimism for the future, reassurance, and support for continued life participation. Similarly to recent statements by others, we recognize the urgent need for and strongly recommend well-conducted clinical studies to evaluate the effects of cannabinoid molecules in patients with rheumatic diseases $^{21}$.

\author{
MARY-ANN FITZCHARLES, MD, \\ Division of Rheumatology, \\ McGill University Health Centre, and \\ Alan and Louise Edwards Pain Management Center, \\ McGill University Health Centre; \\ ELIZABETH M. HAZEL, MD, \\ Division of Rheumatology, \\ McGill University Health Centre, \\ Montreal, Quebec, Canada; \\ GREGORY TAYLOR, MSW, \\ Mary Pack Arthritis Program, \\ Vancouver General Hospital; \\ LORI B. TUCKER, MD, \\ Division of Rheumatology, \\ BC Children's Hospital, \\ University of British Columbia, \\ Vancouver, British Columbia, Canada.
}

Address correspondence to Dr. M.A. Fitzcharles, Montreal General Hospital, 1650 Cedar Ave., Montreal, Quebec H3G 1A4, Canada.

E-mail: mary-ann.fitzcharles@muhc.mcgill.ca

\section{REFERENCES}

1. Goldenberg DL, Clauw DJ, Fitzcharles MA. New concepts in pain research and pain management of the rheumatic diseases. Semin Arthritis Rheum 2011;41:319-34.

2. Volkow ND, Compton WM, Weiss SR. Adverse health effects of marijuana use. N Engl J Med 2014;371:879.

3. Fitzcharles MA, Clauw DJ, Ste-Marie PA, Shir Y. The dilemma of medical marijuana use by rheumatology patients. Arthritis Care Res 2014;66:797-801.

4. Mensinga TT, de Vries I, Kruidenier M, Hunault CC, van den Hengel-Koot IS, Fijen JW, et al. [A double-blind, randomized, placebo controlled, cross-over study on the pharmacokinetics and effects of cannabis.] [Article in Dutch] Nationaal Vergiftigingen Informatie Centrum. RIVM report 267002002/2006. [Internet. Accessed January 15, 2016.] Available from: www.rivm.nl/ dsresource?objectid=rivmp: $12155 \&$ type $=$ org $\&$ disposition $=$ inline\&ns_nc=1\}
5. Asbridge M, Hayden JA, Cartwright JL. Acute cannabis consumption and motor vehicle collision risk: systematic review of observational studies and meta-analysis. BMJ 2012;344:e536.

6. Greydanus DE, Hawver EK, Greydanus MM, Merrick J. Marijuana: current concepts $(\dagger)$. Front Public Health 2013;1:42.

7. Jouanjus E, Lapeyre-Mestre M, Micallef J. Cannabis use: signal of increasing risk of serious cardiovascular disorders. J Am Heart Assoc 2014;3:e000638.

8. Ramesh D, Schlosburg JE, Wiebelhaus JM, Lichtman AH. Marijuana dependence: not just smoke and mirrors. ILAR J 2011;52:295-308.

9. Dosenbach NU, Nardos B, Cohen AL, Fair DA, Power JD, Church JA, et al. Prediction of individual brain maturity using fMRI. Science 2010;329:1358-61.

10. Harkany T, Keimpema E, Barabas K, Mulder J. Endocannabinoid functions controlling neuronal specification during brain development. Mol Cell Endocrinol 2008;286:S84-90.

11. Meier MH, Caspi A, Ambler A, Harrington H, Houts R, Keefe RS et al. Persistent cannabis users show neuropsychological decline from childhood to midlife. Proc Natl Acad Sci U S A 2012;109:E2657-64.

12. Taylor DR, Hall W. Respiratory health effects of cannabis: position statement of the Thoracic Society of Australia and New Zealand. Intern Med J 2003;33:310-3.

13. Zhang LR, Morgenstern H, Greenland S, Chang SC, Lazarus P, Teare MD, et al. Cannabis smoking and lung cancer risk: Pooled analysis in the International Lung Cancer Consortium. Int J Cancer 2015;136:894-903.

14. Callaghan RC, Allebeck P, Sidorchuk A. Marijuana use and risk of lung cancer: a 40-year cohort study. Cancer Causes Control 2013;24:1811-20.

15. Chen CY, Wagner FA, Anthony JC. Marijuana use and the risk of Major Depressive Episode. Epidemiological evidence from the United States National Comorbidity Survey. Soc Psychiatry Psychiatr Epidemiol 2002;37:199-206.

16. Zammit S, Allebeck P, Andreasson S, Lundberg I, Lewis G. Self reported cannabis use as a risk factor for schizophrenia in Swedish conscripts of 1969: historical cohort study. BMJ 2002;325:1199.

17. Gilman JM, Kuster JK, Lee S, Lee MJ, Kim BW, Makris N, et al. Cannabis use is quantitatively associated with nucleus accumbens and amygdala abnormalities in young adult recreational users. J Neurosci 2014;34:5529-38.

18. Lopez-Quintero C, Perez de los Cobos J, Hasin DS, Okuda M, Wang S, Grant BF, et al. Probability and predictors of transition from first use to dependence on nicotine, alcohol, cannabis, and cocaine: results of the National Epidemiologic Survey on Alcohol and Related Conditions (NESARC). Drug Alcohol Depend 2011;115:120-30.

19. Knight JR, Sherritt L, Shrier LA, Harris SK, Chang G. Validity of the CRAFFT substance abuse screening test among adolescent clinic patients. Arch Pediatr Adolesc Med 2002;156:607-14.

20. Fitzcharles MA, Ste-Marie PA, Clauw DJ, Jamal S, Karsh J, LeClercq S, et al. Rheumatologists lack confidence in their knowledge of cannabinoids pertaining to the management of rheumatic complaints. BMC Musculoskelet Disord 2014;15:258.

21. D'Souza DC, Ranganathan M. Medical marijuana: is the cart before the horse? JAMA 2015;313:2431-2.

J Rheumatol 2016;43:686-8; doi:10.3899/jrheum.150103

Personal non-commercial use only. The Journal of Rheumatology Copyright @ 2016. All rights reserved. 